\title{
The New Industrialized Approach to Biology
}

\author{
Review of: Modules in Emerging Fields. Volume 4: Genomics and Proteomics, \\ by Lucienne Ronco, Martha Grossel, Marc Zimmer, and Thomas Socash; CD-ROM
}

\author{
Reviewed by Alan D. Attie, University of Wisconsin-Madison
}

Submitted February 4, 2003; Accepted April 17, 2003

Monitoring Editor: Jeff Hardin

In 1988, the Public Broadcasting System's television program, NOVA, produced a program about the Human Genome Project, "Decoding the Book of Life." At that time, the project was highly controversial. Many researchers were concerned with its high cost and with its potential to distort the priorities of the entire NIH research portfolio. One of its greatest champions was Eric Lander. On the NOVA program, he pulls a book from a shelf and describes how the genome sequence would be each persons "Book of Life." At around the same time, Walter Gilbert predicted that someone would soon be able to pull out a CD and say, "Here is a human being; it's me!" Opposition to the Genome Project evaporated when technological and computational improvements reduced the cost and accelerated the pace of the project. The information that emerged has revolutionized all of biological science.

Enmeshed in this scientific revolution is a cultural revolution that manifests itself in several ways.

1. Biology adopted the "Big Science" model. There are now many genome projects, genome centers, and large-scale projects to extend the fruits of the genome projects-e.g., large-scale mutagenesis in model organisms, proteomics. The computing requirements of biology now exceed the computing requirements of physics.

2. Great discoveries in biology can come without a hypothesis or a clever insight. The systematic application of industrial-scale programs has delivered novel genes and novel biological information. For example, the ability to mutagenize the entire genome of the model nematode C. elegans with siRNA has led to profound findings related to aging, although the actual design of the experiments simply consists of a mutagenesis screen for longevity.

3. The ready access to genomic information has made most biologists into reductionists. The functional unit of analysis in biology is the gene or the protein. The ability to do

DOI: $10.1187 /$ cbe.03-02-0008

Corresponding reviewer. E-mail address: attie@biochem.wisc. edu.

Note. This CD-ROM was produced by Connecticut College with support from the Howard Hughes Medical Institute and the W.M. Keck Foundation. The CD-ROM is available free of charge to instructors. The CD contains three seminars, brief video clips, PowerPoint presentations, and a collection of links to Web resources in genomics, proteomics, and bioinformatics. Interested instructors should send a self-addressed-stamped envelope to Dr. Grossel to receive one free copy. Mail to: Martha J. Grossel, Box 5331, Connecticut College, 270 Mohegan Avenue, New London, CT 06320. forward and reverse genetics (screen for a gene based on phenotype or knock out a gene and then study phenotype) makes a gene-centered approach to biological problems extremely powerful and effective.

I believe that in the future this CD-ROM will be an interesting time capsule that captures a phase in an ongoing scientific and cultural revolution.

The module consists of

- a seminar by Craig Venter, founder of Celera, Inc.;

- two pedagogical seminars- "The Dynamic Genome: The New Industrialized Approach to the Study of Biology," by Lucienne Ronco (40 min), and "The Dynamic proteome: Translating Industrialized Biology to Proteins," by Josh LaBaer (60 min).

- PowerPoint files containing the slides of the two Ronco and LaBaer seminars;

- a video tour of the robotics and research lab at the Harvard Institute of Proteomics (40 min); and

- three short question/answer modules ( $<5 \mathrm{~min}$ ), with Craig Venter commenting on gene patenting, human cloning, and computing.

Venter gives a historical and autobiographical account of his entrée into sequencing. He describes how Perkin-Elmer, Inc., approached him with an unsolicited \$350 million to create Celera (he hung up the phone the first two times because he thought it was a crank phone call). Venter describes how his approach to sequencing the genome was met with skepticism; it involved fragmenting the genome and sequencing the fragments, then reassembling the sequence. The public consortium took the slower but more systematic approach of sequencing bacterial artificial chromosome (BAC) clones. With laudable diffidence, Venter tells us that his approach succeeded, but the crucial breakthrough came from computing, not sequencing; without novel algorithms and faster supercomputers, it would have been impossible to assemble the genomes in a timely fashion. The reductionist creed creeps through in several places. Venter tells us that we need a computer to know "how to build" an organism.

Lucienne Ronco's seminar has some overlap with Venter's. It gives an historical overview of the genome projects and some of the results. It goes into details that could have been edited (e.g., showing a list of genome centers). The discussion of RNA quantitation methods is a bit tedious for an overview talk. Conversely, the overview of some genetics 
topics (e.g., linkage disequilibrium) is probably not going to be understandable to most undergraduates without additional background material. The list of ethical issues is quite good and a helpful starting point for instructors to use for classroom discussion.

Josh LaBaer gives a lucid description of methods for analyzing proteins. This includes 2-D electrophoresis and mass spectrometry. He summarizes the kinds of questions that one asks in order to try to ascertain the functions of proteins (e.g., cell location, interacting proteins, structural motifs). Consistent with the technological orientation of this module, LaBaer discusses the challenges that come with scale-up. He shows how his laboratory is doing high-throughput cloning and expression of human genes. Much of the discussion focuses on the clever engineering tricks employed to automate the cloning steps (e.g., a robotic clone picker equipped with a camera and toothpicks). He describes how high-throughput gene expression in mammalian cells can be used for phenotype screens. He demonstrates how a screen for genes that cause cells to migrate through a membrane can be used to identify potential targets for drugs aimed at preventing metastasis. The video unfortunately cuts off LaBaer before he completes a discussion of a clever comprehensive bibliographic search scheme to identify genes likely to be implicated in a multitude of diseases.

Both Ronco's and LaBaer's seminars are good reserve material for biochemistry/biotechnology courses. Since most experienced instructors do not require somebody else's narration, they may wish to make use of the excellent PowerPoint slides that are included in the package.

In a 5-min Q\&A on gene patenting, Venter glibly states that "it's much ado about nothing." He states the importance of granting exclusivity as an incentive for companies to invest the great resources required for product development, a subject that was never at issue in the gene patenting debates. Venter is hardly a neutral observer; he tested the limits of gene patenting by filing thousands of patents on sequences, many of them expressed sequence tags (ESTs). Patenting is still a hot and contentious topic. If gene patenting is to be presented to students, the many relevant issues should be aired by somebody who does not have a stake in one particular position.

On human cloning, Venter correctly states that it already occurs naturally through twinning and that many foolish people think that they can recreate the same "person" by cloning that person. But, he misses a "teaching moment" by not going into many of the biological issues (e.g., senescence, mitochondrial DNA, imprinting) or ethical issues (cloning for stem cells vs. cloning to produce humans). Instead, he derisively describes an encounter with several priests he debated at a conference in Spain who made a naive distinction between human genes and nonhuman genes. This trivializes a topic that could be discussed on a much higher plane.

A shortcoming of this module is that it did not go beyond the practitioners for an historical, philosophical, and ethical perspective. The Genome Project funded numerous outstanding scholars in these other disciplines under the "ELSI" program (Ethical, Legal, and Social Implications of genetics research). It is unfortunate that most biology students are not exposed to this valuable body of scholarship. Although it is interesting from an historical perspective to have Craig Venter answer some of the ethical questions, it is analogous to studying a war by speaking only to the soldiers. None of the listed URLs include the ELSI program or the many organizations and societies devoted to the study of the historical, philosophical, and ethical issues surrounding this scientific revolution.

The lab tour consists of nine short videos of the robotics laboratory at the Harvard Institute of Proteomics, some techniques, and their automation (PCR, electrophoresis, bacterial transformation, plasmid purification, DNA sequencing). The videos are narrated by lab personnel in an informal style. They give the viewer a realistic feel for the lab and some of the methods. For students familiar with the methods and interested in the way the methods are developed for high throughput, this will be especially interesting.

The URL list is surprisingly short and narrow; it consists primarily of NIH-based genome sites. Missing from the list are numerous other important data analysis/retrieval sites (e.g., the excellent UC-Santa Cruz "Golden Path" site: http: / / genome.ucsc.edu/) and a tremendous array excellent of educational sites and resources.

This module made me wonder if genome-based, technologically driven biology is going to displace hypothesis-driven research. Not likely; there are still many highly creative and insightful biologists who continue to hit the mark with great ideas. But, for educators, it is much harder to teach students about this process than it is to expose them to powerful technology and its dramatic results. I hope that somebody follows this technology module with a CD-ROM dedicated to great ideas in biology and how they were conceived and validated. 